\title{
La transición al pos-productivismo: parques patrimoniales, parques culturales y ordenación territorial
}

Pablo Alonso. Universidad de Cambridge, Cambridge, Reino Unido.

RESUMEN | El fenómeno de los parques patrimoniales se está propagando en Estados Unidos y Europa y se extiende por otras áreas a medida que el sector terciario gana peso en la economía y las dimensiones de la cultura y el ocio tienden a hibridarse. Los parques patrimoniales son iniciativas heterogéneas que colocan el patrimonio cultural en el centro de las políticas de ordenación territorial y desarrollo económico, buscando la reinvención de territorios amplios y la participación de las comunidades locales en el planeamiento. Su relevancia deriva de su potencial influencia en la configuración macroterritorial de regiones amplias y en la articulación de las áreas protegidas tradicionales. Pese a ello, han recibido una escasa atención académica hasta el momento. Este trabajo expone los resultados de una investigación realizada a partir del estudio de la literatura disponible y busca la apertura del asunto a la interpelación académica, tanto de las disciplinas técnicas como sociales.

PALABRAS CLAVE | transformaciones socioterritoriales, patrimonio, gestión territorial.

ABSTRACT | The development of heritage area is an increasing trend in the United States and Europe, a phenomenon spreading over other areas where the tertiary economic sector is gaining weight and the dimensions of culture and leisure tend to hybridize. Heritage areas or parks are heterogeneous initiatives that place cultural heritage at the heart of spatial planning policy and economic development, aiming at the reinvention of large territories and local community participation in planning. Their relevance stems from their potential influence on the territorial configuration of broad regions and their impact upon the articulation of traditional protected areas. Notwithstanding this, they have attracted scant academic attention so far. This paper presents the results of an investigation that has analyzed most available literature on the topic, with the aim to open the issue of heritage areas to academic scrutiny of both technical and social disciplines.

KEY WORDS | socio-territorial transformations, heritage, territorial management.

Recibido el 4 de enero de 2012, aprobado el 16 de agosto de 2012

E-mail: Pa332@cam.ac.uk

El artículo sintetiza los resultados de la tesis de Maestría “Projecting the past, preserving the future: What is a Cultural Park?”, realizada en la Universidad de Cambridge (2010-2011), y se encuadra dentro de los siguientes proyectos de investigación: HAR2010-21950-C03 "La formación de los paisajes del NO peninsular durante la Edad Media (siglos V-XII), Ministerio de Ciencia e Innovación de Espańa; y LE302A11-1 "León en la Alta Edad Media. El territorio de Cea entre los siglos V-XII, Junta de Castilla y León (Espańa).

Agradecimientos a Joaquím Sabaté Bel y Margarita Fernández Mier. 


\title{
Introducción
}

\begin{abstract}
Incluso dentro de los grupos de preservación histórica y gestión de parques hay un desconocimiento generalizado de los Parques Patrimoniales. (...) Estos no se ajustan a ningún concepto o especialización común. (...) Y el planeamiento, desarrollo y gestión de los mismos requiere la coordinación de varias habilidades especializadas -incluyendo las de arquitectos, arquitectos paisajisticos, planeadores territoriales, restauradores, pedagogos, economistas y especialistas en turismo- para dar cuenta de las intrincadas relaciones que existen en un paisaje viviente dentro de un parque patrimonial. Una consecuencia positiva es la oportunidad de ampliar las posibilidades de los diferentes especialistas a través de la vinculación entre distintas disciplinas. Pero esta situación ha dejado a los parques patrimoniales huérfanos, sin una profesión especializada que pueda considerarlos dentro de su ámbito de acción (Bray, 1994, p. 34).
\end{abstract}

Bray captura la esencia del problema que la investigación afronta al plantearse la cuestión de las áreas o parques patrimoniales o culturales ${ }^{2}$. Podría decirse que estos han sido encerrados en un círculo aislado que se retroalimenta: prácticamente no se los ha estudiado, por su carácter complejo e interdisciplinar, y esta misma índole dificulta el avance de la investigación. Esto es así, porque forman parte de un tipo de entidades que Law y Mol (2002) denominan resbaladizas o elusivas, ya que obstaculizan los principios de la investigación científica, basada en la individuación clara y concisa de elementos o áreas delimitadas para su análisis. Así, resulta imposible intentar aprehender los parques patrimoniales exclusivamente desde el punto de vista de la ordenación del territorio, ya que inevitablemente hay que remitirse a otros aspectos que entran de lleno en el campo de acción de las ciencias sociales, e incluso humanas. Sin renunciar a esta necesaria interdisciplinariedad, planteamos en este artículo una visión general de los parques patrimoniales en su dimensión de proyectos territoriales. A modo introductorio, podemos concebirlos como proyectos a escala regional que funcionan a través de la cooperación de diversos actores sociales a distintas escalas y que buscan el desarrollo económico a partir de la puesta en valor de elementos patrimoniales, el refuerzo de la identidad local y la creación de una marca de cara al turismo, sin por ello controlar los usos del suelo o su propiedad.

El fenómeno de los parques patrimoniales se ha extendido con mucha fuerza en tiempos recientes y especialmente en los centros neurálgicos de la economía mundial, como son Europa y Estados Unidos. En el resto del mundo su difusión es creciente. En Asia existe una mayor preocupación por la protección de los paisajes culturales que por su gestión, debido al potente desarrollismo industrial en el área (Taylor, 2009). En Latinoamérica se produce una situación similar, pero existe una mayor preocupación y concienciación respecto de la protección y gestión de los

1 Las citas que no cuentan con una versión en español han sido traducidas directamente del inglés por el autor.

2 Aquí usaremos consistentemente "parque patrimonial". 
paisajes culturales (Sabaté, 2010). En todo caso, es fácil prever la extensión de este tipo de iniciativas en el área, dado el carácter generalmente disperso del patrimonio y el elevado ritmo de desarrollo económico y social actual en la región.

Esta realidad plantea varios problemas a la ordenación territorial. ¿Cómo planificar un territorio a partir de un intangible como es la identidad? ¿Cómo mantener un equilibrio entre la preservación del pasado y la proyección del futuro, entre el patrimonio como cultura y como producto de ocio, o entre la visión del territorio de las comunidades locales y los turistas? ¿Hasta dónde ha de llegar la intervención y artificialización del territorio? O, dicho de otro modo, ¿cómo mantener un equilibrio entre la musealización del mismo y su conversión en un parque temático? En resumen, ¿cómo traducir la identidad del paisaje a la ordenación y gestión territorial?

La hipótesis de partida es que el fenómeno de los parques patrimoniales se ha extendido debido a transformaciones socioeconómicas estructurales en las áreas más desarrolladas, ocurridas en torno a dos ejes: la terciarización de la economía y la centralidad del concepto de patrimonio, que sirve como enlace a cuestiones emocionales, afectivas e identitarias y hace que resulte necesario el establecimiento de un diálogo entre las ciencias sociales y la ordenación territorial. Esta es una tarea compleja, ya que para comprender el funcionamiento de un parque o para ponerlo en marcha son necesarias las herramientas de la antropología/etnografía, la economía, la arquitectura, la historia, los estudios de patrimonio y los de turismo, como poco. Por ello, resulta difícil disgregar aspectos técnicos de proyección y gestión de la dimensión sociocultural del parque, ya que estos fomentan la participación de las comunidades locales a la vez que se nutren del patrimonio cultural de las mismas como valor territorial que explotar turísticamente. Así, consideramos importante mantener un enfoque simétrico en el análisis de los parques patrimoniales, de modo que no sean considerados simplemente como instrumentos técnicos o, en el otro extremo, como "construcciones sociales" exclusivamente (Latour, 1992).

Los datos fundamentales para el desarrollo de nuestro argumento provienen de un análisis pormenorizado de la bibliografía existente, tanto impresa como en formato digital. A esto se añade nuestra propia experiencia en contextos de gestión e intercambios, tanto formales como informales, con especialistas en el ámbito. El objetivo esencial de este estudio no es llevar a cabo una síntesis histórica ni una clasificación de los parques patrimoniales, algo que puede encontrarse en otros trabajos (Bustamante \& Ponce, 2004; Sabaté, 2004), sino más bien abrir la cuestión de los mismos al escrutinio académico; no solo a la indagación desde la ordenación territorial, sino también a la interpelación de las ciencias sociales. Se trata, por tanto, de una reflexión teórica con intención de enriquecer el funcionamiento de los parques en contextos prácticos. Para ello, ofrecemos una somera visión de las transformaciones territoriales contemporáneas en las que se enmarca la creación de parques patrimoniales. Posteriormente analizaremos las raíces epistemológicas de los mismos a partir del concepto de paisaje, pasando a una sintética exposición del funcionamiento de los parques en EE.UU. y Europa. Finalmente, se debaten cuestiones relevantes para la comprensión y discusión del fenómeno de los parques patrimoniales. 


\section{Nuevas soluciones para nuevos problemas: el paso al pos-productivismo}

Comprender las transformaciones que el productivismo funcionalista había inducido en las formas de ver y gobernar el territorio es esencial para entender el porqué de los parques patrimoniales. La organización del territorio y la población en torno a las labores productivas/extractivas llevó a una desterritorialización o ruptura de la relación entre el territorio y el entorno antrópico. A cada territorio le venía adscrita una función con el objetivo de maximizar el valor extraíble de cada configuración territorial (Rullani, 2006). El progreso técnico permitía liberar la ordenación del territorio de los condicionantes físicos. Así, se desvinculaba al ser humano del territorio que había construido a lo largo del tiempo. Este proceso de homogeneización territorial ha sido concebido por Augé (2008) como la expansión de los "no-lugares", o junk space según Koolhaas (2002). Casey (1997) y Choay (2007) observan la constante disolución de los elementos constitutivos de la identidad y complejidad del territorio ante una forma de ordenación que pasa por alto los factores distintivos locales.

Las últimas dos décadas han presenciado la transición de vastas regiones de EE.UU. y Europa hacia sistemas pos-productivistas que han supuesto una transformación del rol del territorio (Fumagalli, 2007). El proceso de deslocalización del centro a la periferia lleva a la desindustrialización de amplias áreas, exacerbando la ruptura entre la ciudad y el territorio, y las jerarquías territoriales (Marazzi, 2010). Se plantea entonces la cuestión de qué hacer con estos territorios empobrecidos. La respuesta habitual es relegarlos a un papel subordinado, donde han de soportar funciones destructivas o contaminantes. También es posible sostener de forma artificial sus economías mediante subsidios a la agricultura, lo que ocurre tanto en EE.UU. como en Europa, o a través de proyectos de desarrollo rural y cohesión territorial, como los desarrollados en Europa.

Para algunos territorios se ha abierto otra puerta a partir de lo que algunos autores consideran el matiz diferencial del pos-productivismo: el devenir renta del beneficio. Negri y Vercellone (2007) consideran renta la ganancia de un propietario por el mero hecho de poseer algo, acepción tradicionalmente utilizada en el mercado inmobiliario y en el de las tierras agrarias. Por el contrario, entienden beneficio como algo productivo basado en la capacidad de generar una plusvalía a partir del valor añadido del producto y del trabajo de la mano de obra. Podemos así hablar de "rentas territoriales" generadas en la intersección entre turismo, patrimonio y paisajes distintivos. Este proceso es lo que Rullani (2009) denomina la "repersonalización" de la economía y del territorio, que supone una vuelta a la centralidad de los lugares, espacios asociados a identidades no ya dadas, sino producidas por la gente a partir de la construcción de significados culturales. Se busca así la diferenciación de cada territorio basándose en la creación de un doble vínculo: uno local, ligado a la tradición del lugar; otro global, que entra en los flujos de distribución de valores y deseos.

En la práctica, lo anterior se plasma en la entrada en la competencia territorial global por atraer inversiones y turismo, donde la acumulación de capital simbólico territorial resulta esencial. Esta capacidad de producir lugares ha llevado a muchas regiones desindustrializadas europeas y estadounidenses a reinventarse a partir de las rentas territoriales que el binomio patrimonio-turismo cultural permite sustentar, 
procurando que funcione como economía de arrastre para con el resto de sectores. Así, podemos concebir los parques patrimoniales como instrumentos de ordenación y gestión de la renta territorial que una determinada área ostenta y produce.

\section{Hibridación entre territorio y patrimonio: los paisajes culturales}

El concepto de paisaje cultural se ha convertido en acepción de uso corriente en instituciones como la Organización de las Naciones Unidas para la Educación, la Ciencia y la Cultura (Unesco, por United Nations Educational, Scientific and Cultural Organization) o el Servicio de Parques Nacionales (National Park Service, NPS) de los EE.uU. Doménech (2005) define paisaje cultural como:

El resultado de una continua y gradual sedimentación de procesos socioeconómicos que reflejan la evolución de una sociedad en un territorio. No es posible discutir sobre paisaje, arte o patrimonio sin tener en cuenta el protagonismo del hombre en el territorio. El ser humano es el actor y agente de las transformaciones que dejan trazas en el paisaje (p. 64).

La utilidad del concepto radica en que permite la generación de "diferencias" y, consecuentemente, de valor territorial. Así, paisajes culturales son paisajes diferentes del paisaje en general; son únicos, excepcionales y bellos. De esta forma se vincula paisaje con la idea de patrimonio, algo común que debe ser preservado en su vertiente de identidad social y puesto en valor desde un punto de vista utilitarista, como recurso con valor de uso directo.

La hibridación de paisaje y patrimonio sustentará la mayoría de parques patrimoniales europeos, siguiendo la visión de la Unesco, para la cual los paisajes culturales:

Son bienes culturales y representan las "obras" conjuntas del hombre y la naturaleza (...). Ilustran la evolución de la sociedad humana y sus asentamientos a lo largo del tiempo, condicionados por las limitaciones y/o oportunidades físicas que presenta su entorno natural y por las sucesivas fuerzas sociales, económicas y culturales, tanto externas como internas (Unesco, 2005, pár. 47, p. 48).

En los EE.UU., la definición más extendida es la del NPS, que considera el paisaje cultural como "un área geográfica, incluyendo los recursos culturales y naturales y los animales salvajes y domésticos presentes en ella, asociada con un evento, actividad o personaje histórico o que presenta otros valores estéticos o culturales" (Birnbaum \& Capelle, 1996, p. 4).

\section{Parques culturales: definiciones y funcionamiento}

\section{Genealogía}

Los parques patrimoniales no son instrumentos originales, sino más bien un producto híbrido de avances en disciplinas académicas y de trasformaciones en prácticas institucionales, principalmente producidos en EE.UU. y Europa. En Europa resulta esencial la conceptualización del museo (i.e. Prado, Louvre, etcétera) como fuente de legitimidad del Estado-nación surgido en el siglo xIx, idea que ha traído 
consigo una separación radical entre museos y áreas protegidas, como los parques naturales o nacionales.

Igualmente influyente es la tradición escandinava de museos al aire libre donde se exponían colecciones folclóricas en contacto con la naturaleza, como la sección externa del Museo Escandinavo de Etnografía (1873) o el museo Skansen (1891). Esta tradición influenció decisivamente el desarrollo de la Nueva Museología y del Ecomuseo, del que los parques patrimoniales toman su voluntad de convertirse en representantes de la comunidad local, abrirse a territorios amplios y sintetizar las relaciones hombre-naturaleza en el tiempo y el espacio. Destaca la tradición italiana de ordenación de espacios protegidos, con un marcado carácter culturalista en contraste con la perspectiva naturalista-funcionalista estadounidense. Un programa similar es el sistema francés de parques regionales, donde se incluyen formas de propiedad y actores diversos con el objetivo de utilizar los recursos patrimoniales como soporte del desarrollo económico (Lacosta Aragües, 1997).

Finalmente, la Arqueología Industrial contribuyó al replanteamiento del concepto de sitio patrimonial, al concebirlo siempre como un paisaje amplio, acercando el patrimonio a las comunidades locales y vinculándolo al desarrollo económico gracias a los grupos de acción local. Proyectos nacidos en los años sesenta, como Ironbridge, New Lanark o Le Creusot-les-Mines, abrieron la puerta al nacimiento de los parques patrimoniales. Por su parte, el sistema de Parques Nacionales estadounidense comenzó con Yellowstone (1872) y se institucionalizó con el establecimiento del NPS en 1916. Los parques desempeñan un papel similar al de los museos europeos como "mitos pastorales" donde descansa la identidad nacional (Bray, 1994).

La creación del National Trust for Historic Preservation [Fundación Nacional para la Conservación Histórica] (1949) y la sanción del National Historic Preservation Act [Ley Nacional para la Conservación Histórica] (1966) abrieron la puerta al surgimiento de los parques patrimoniales estadounidenses o National Heritage Areas (NHA) (Eugster, 2003). Otros hitos relevantes en la década de 1960 fueron el giro ambientalista, el nacimiento de la escuela de planeamiento ecológico y el desarrollo de la arqueología industrial. Durante los años ochenta destacan el nacimiento de iniciativas de gestión espacial y patrimonial en varios estados, y en particular el sistema de Parques Culturales Urbanos del estado de Nueva York. Este programa buscaba la participación de las comunidades locales y la consecución de objetivos económicos y no solo de preservación.

Estas iniciativas condujeron al replanteamiento de la posibilidad de gestionar unilateralmente las "áreas protegidas", algo que según Nelson y Sportza (2000) resultaba insostenible. Así, el documento National parks for a new generation: visions, realities, prospects (Conservation Foundation, 1985) reconocía que los proyectos locales y regionales que afloraban por todo el país eran de hecho parques y debían ser reconocidos oficialmente. Esto implicaba que el NPS debía ofrecer a estos proyectos el soporte técnico y económico que les había negado hasta la fecha. Este nuevo contexto abría la puerta al nacimiento de las NHA en los EE.UU. 


\section{Estado de la cuestión}

Resulta sorprendente la escasa atención que los parques patrimoniales han recibido en ámbitos académicos. Geográficamente, los estudios provienen de Europa y EE.UU., áreas donde el fenómeno se ha desarrollado, aunque existen excepciones (Henry, 2000; Wang \& Sun, 2001). A nivel disciplinar, es claro el predominio de visiones técnicas desde la arquitectura y la ordenación espacial, con la ausencia notable de análisis desde las ciencias sociales/humanidades, a excepción de algunos estudios geográficos.

Nuestra investigación considera que esta preeminencia de visiones técnicas se debe en parte a la reticencia de las ciencias sociales/humanidades a involucrarse en cuestiones pragmáticas e, inversamente, a la necesidad de arquitectos y planeadores de enfrentarse en la práctica con nuevas problemáticas socioterritoriales. También su carácter interdisciplinario y complejo ha obstaculizado su investigación: en un parque patrimonial no se pueden desligar o "purificar" conceptos para su análisis individualizado, ya que los objetos de estudio del turismo, ordenación territorial, patrimonio, museología, arquitectura o historia se encuentran entremezclados. Finalmente, su carácter dinámico y variable dificulta la tarea científica de "fijar", estabilizar y generalizar funciones y significados.

\section{Centrando el concepto: distinciones y definiciones}

Para la realización de este apartado nos basaremos en estudios cualitativos, ya que a nivel cuantitativo los análisis son inexistentes. Pese a ello, la emergencia del fenómeno se confirma cuando analizamos los datos de países como España o EE.uU., donde el número de parques naturales o nacionales prácticamente no ha aumentado, mientras que el de parques culturales o patrimoniales crece constantemente. Así, desde 1980 se han creado en EE.UU. solo diez parques nacionales por cincuenta y cuatro áreas patrimoniales (Cuadro 1). Los fundamentos tradicionales de los parques nacionales, reservas de la biosfera o parques naturales en sus múltiples acepciones a escala internacional respondían a un paradigma clásico consistente en el binomio naturaleza y conservación. El objetivo era delimitar espacios y extirparlos de su contexto social como "islas" para proteger la espectacularidad de ciertos panoramas y la vida salvaje. Eran, además, vistos como cuestiones de interés para la nación, no para la comunidad, y financiados con fondos públicos. El control gubernamental del suelo implicaba una gestión técnico-burocrática y científica contra las personas, cuya opinión e incluso formas de vida eran escasamente tenidas en cuenta (Phillips, 2002).

\section{CUADro 1 | "National Heritage Areas" en EE.Uu. y año de declaración}

\begin{tabular}{|c|l|}
\hline AÑo & \multicolumn{1}{|c|}{ NOMBRE Y ESTADO } \\
\hline 1984 & Illinois \& Michigan Canal National Heritage Corridor, IL \\
\hline 1986 & John H. Chafee Blackstone River Valley National Heritage Corridor, MA \\
\hline 1988 & Delaware \& Lehigh National Heritage Corridor, PA \\
\hline 1988 & Southwestern Pennsylvania Heritage Route (Path of Progress), PA \\
\hline 1994 & Cane River National Heritage Area, LA \\
\hline
\end{tabular}




\begin{tabular}{|c|c|}
\hline AÑo & NOMBRE Y ESTADO \\
\hline 1994 & Quinebaug \& Shetucket Rivers Valley National Heritage Corridor, Ст \& MA \\
\hline 1996 & Cache La Poudre River Corridor, co \\
\hline 1996 & America’s Agricultural Heritage Partnership, IA \\
\hline 1996 & Augusta Canal National Heritage Area, GA \\
\hline 1996 & Essex National Heritage Area, NY \\
\hline 1996 & Hudson River Valley National Heritage Area, MA \\
\hline 1996 & National Coal Heritage Area, wv \\
\hline 1996 & Ohio \& Erie Canal National Heritage Corridor, он \\
\hline 1996 & Rivers of Steel National Heritage Area, PA \\
\hline 1996 & Shenandoah Valley Battlefields National Historic District, va \\
\hline 1996 & South Carolina National Heritage Corridor, sc \\
\hline 1996 & Tennessee Civil War Heritage Area, TN \\
\hline 1998 & Automobile National Heritage Area, MI \\
\hline 2000 & Wheeling National Heritage Area, wv \\
\hline 2000 & Yuma Crossing National Heritage Area, AZ \\
\hline 2000 & Lackawanna Valley National Heritage Area, PA \\
\hline 2000 & Schuylkill River Valley National Heritage Area, PA \\
\hline 2000 & Erie Canalway National Heritage Area, NY \\
\hline 2003 & Blue Ridge National Heritage Area, NC \\
\hline 2004 & Mississippi Golf Coast National Heritage Area, Ms \\
\hline 2004 & National Aviation Heritage Area, OH \\
\hline 2004 & Oil Region National Heritage Area, PA \\
\hline 2006 & Arabia Mountain National Heritage Area, GA \\
\hline 2006 & Atchafalaya National Heritage Area, LA \\
\hline 2006 & Champlain Valley National Heritage Partnership, NY \& VT \\
\hline 2006 & Crossroads of the American Revolution National Heritage, Area NJ \\
\hline 2006 & Freedom's Frontier National Heritage Area, кs \& MO \\
\hline 2006 & Great Basin National Heritage Area, NV \& UT \\
\hline 2006 & Gullah/Geechee Cultural Heritage Corridor, NC, sC, GA, FL \\
\hline 2006 & National Mormon Pioneer Heritage Area, uT \\
\hline 2006 & Northern Rio Grande National Heritage Area, NM \\
\hline 2006 & National Housatonic Valley National Heritage Area, ст \& мА \\
\hline 2008 & Abraham Lincoln National Heritage Area, PL \\
\hline 2008 & Journey through Hallowed Ground National Heritage Area, PL \\
\hline 2008 & Niagara Falls National Heritage Area, NY \\
\hline 2009 & Susquehanna Gateway National Heritage Area., PA \\
\hline
\end{tabular}


(continuación)

\begin{tabular}{|l|l|}
\hline AÑo & \multicolumn{1}{|c|}{ NOMBRE Y ESTADO } \\
\hline 2009 & Santa Cruz Valley National Heritage Area, AZ \\
\hline 2009 & Sangre de Cristo National Heritage Area, CO \\
\hline 2009 & South Park National Heritage Area, CO \\
\hline 2009 & Northern Plains National Heritage Area, ND \\
\hline 2009 & Baltimore National Heritage Area, MD \\
\hline 2009 & Freedom's Way National Heritage Area, MA/NH \\
\hline 2009 & Mississippi Hills National Heritage Area, MS \\
\hline 2009 & Mississippi Delta National Heritage Area, Ms \\
\hline 2009 & Muscle Shoals National Heritage Area, AL \\
\hline 2009 & Kenai Mountains-Turnagain Arm National Heritage Area, AK \\
\hline 2011 & Sacramento-San Joaquín Delta National Heritage Area, CA \\
\hline 2011 & Buffalo Bayou National Heritage Area, Tx \\
\hline
\end{tabular}

FUENTE ELABORACIÓN PROPIA

Las áreas patrimoniales buscan diferenciarse de este paradigma de gestión que todavía resulta mayoritario internacionalmente. En primer lugar, el objetivo de las áreas patrimoniales no es la preservación, sino la gestión activa de recursos culturales -aunque también incluyen recursos naturales ocasionalmente- de cara a la promoción de la identidad local y el desarrollo económico (Bray, 1994). A diferencia de otros modelos de gestión territorial, se suelen organizar de abajo hacia arriba a partir de grupos de acción local. Las comunidades crean así redes de conexiones o "partenariados" donde participa toda clase de agentes sociales y no solo instituciones públicas. Tanto en Europa como en EE.UU. esto se suele traducir en que la preservación de los recursos naturales y culturales se realice a nivel cooperativo local, sin necesidad de que ninguna institución -tradicionalmente el NPS en EE.UU. o los estados en Europa o Latinoamérica (Barrett, 2003) - se convierta en propietaria del suelo (Frenchman, 2004). Sin embargo, la mera restitución del control de la gestión de parques o reservas a las comunidades locales, como en los casos de Oaxaca en México, Cofán de Bermejo en Ecuador o el Parque Nacional Indiwasi en Colombia (Oviedo, 2004), no significa necesariamente que aquellos se conviertan en parques patrimoniales.

Los parques patrimoniales generalmente desarrollan planes a largo plazo donde se identifican los objetivos regionales, se marcan estrategias y asignan responsabilidades a los actores sociales. Al contrario que otros parques o reservas, las áreas patrimoniales intervienen activamente en el territorio, estableciendo vínculos entre corredores culturales o naturales, servicios de turismo y espacios culturales. Para su gestión se crean entidades que guían el proceso de implementación, las cuales habitualmente carecen de propiedad o control sobre los usos del suelo (Daly, 2003). Otro factor distintivo de los parques o áreas patrimoniales es "su énfasis en la interacción entre la gente y su entorno. Las áreas patrimoniales buscan contar la historia de la gente a lo largo del tiempo y cómo el paisaje dio forma a las tradiciones de los habitantes" (Vincent \& Whiteman, 2008, p. 1). Así, se suele organizar 
el territorio a partir de paisajes culturales o itinerarios culturales organizados alrededor de algún tema central, como el pasado industrial, episodios bélicos, áreas arqueológicas o similares (Clavel-Lévêque, Doukellis \& Tirologos, 2002). La clave es que el patrimonio funciona como "principio organizador" (Eugster, 2003), desde el cual las comunidades locales planifican su futuro económico, medioambiental y cultural. Las áreas patrimoniales unifican a la comunidad, porque:

Todas las personas tienen un patrimonio que significa algo para ellas (...). Las áreas patrimoniales tienen un corazón, alma y espíritu de los cuales carecen los planes de ordenación tradicional de suelos y del territorio. Las áreas patrimoniales permiten a la gente reclamar estos lugares y dar relevancia a la comunidad, el paisaje y la región (Eugster, 2003, p. 51).

De este modo, el énfasis pasa de lo nacional a lo local, y la gestión de manos de expertos y científicos a agentes e intermediarios que combinan estos conocimientos con el de lo local, desempeñando varias funciones (Phillips, 2003).

Al sustentarse en la idea de paisajes culturales, la aproximación de la sociedad hacia su entorno cambia de un enfoque de gestión técnico-burocrática hacia cuestiones de interpretación. Así, la puesta en valor del paisaje se concibe como la facilitación de un código al visitante foráneo para:

Realzar la significancia cultural del territorio a través de una lectura que valora la memoria (...). Así, la identidad y las fuerzas económicas y socioculturales pueden transformar la memoria en un factor de innovación, en nuevas formas de desarrollo y preservación. Se trata de iniciativas que permiten una metamorfosis de los lugares de modo que recobren fuerza la identidad cultural y el sentido de pertenencia territorial (Barilaro, 2006, p. 101).

Como vemos, estas visiones orientan las transformaciones previamente referidas hacia una repersonalización de la economía, donde lo fundamental es recobrar el alma, la identidad local, el sentido de pertenencia, y garantizar la distinción de cada región a través de procesos de valorización (Rullani, 2006). De este modo, las sinergias territoriales "se refuerzan a través de la narración de las historias de la gente y sus lugares. Estas historias reflejan una identidad regional y fundamentan una sensibilización colectiva sobre la necesidad de valorizar lo que hace único a cada lugar" (Daly, 2003, p. 2).

Así, los parques patrimoniales son ambientes humanizados donde se desarrolla la vida normal de sus habitantes. Pero, a la vez, intentan configurarse como polos de atracción para un tipo de turismo fundamentalmente cultural y de calidad (Conzen, 2001). Es decir, se trata de espacios de ocio y esparcimiento tanto para la población local como para el visitante, en ambientes antropizados y no ya funcionalmente apartados como "verde" en espacios delimitados. Su objetivo último es la transformación de espacios generalmente deprimidos en áreas dinámicas económicamente. Para ello utilizan estrategias de marketing y comercialización que favorecen la construcción de imágenes de marca para identificar el territorio, sus recursos y valores (Bustamante \& Ponce, 2004). Las áreas patrimoniales son intervencionistas y tienen objetivos comerciales, sin ser por ello parques temáticos, 
ya que gestionan identidades y patrimonios reales en aras de la sostenibilidad y no de la intensificación del beneficio (Sabaté \& Lista, 2001).

Las heterogéneas definiciones legislativas y académicas de los parques culturales en Europa resultan de ideas provenientes de la Unesco y el Convenio Europeo del Paisaje (Europa, 2000), donde el paisaje desempeña un papel fundamental. Según la Ley de Parques Culturales de Aragón (1997), un parque patrimonial es “un territorio que contiene elementos relevantes del patrimonio cultural, integrados en un marco físico de valor paisajístico y/o ecológico singular, que gozará de promoción y protección global en su conjunto, con especiales medidas de protección para dichos elementos relevantes" (Cap. 1, art. 1). En cambio, Sabaté (2009) lo concibe como "un instrumento de proyecto y gestión, de reconocimiento y puesta en valor de un determinado paisaje cultural, que persigue no solo la preservación de su patrimonio o la promoción de la educación, sino asimismo favorecer el desarrollo económico local” (p. 626). Para Bergdhal (2005):

El concepto de parque cultural ha ampliado su alcance. (...) El objetivo de su organización no es solo el de preservar la historia de una región. Se trata de contribuir a un desarrollo económico positivo de la misma, objetivo poco usual para un museo, lo cual significa que está proyectándose más hacia el futuro de la región, que hacia su pasado (p. 61).

Por su parte, Bustamante y Ponce (2004) lo consideran "proyecto que privilegia la construcción de una imagen que otorga identidad al territorio, donde el patrimonio y otros recursos culturales y naturales se combinan, exponen, aumentan y promueven intencionadamente" (p. 14).

En EE.UU. es habitual que los propios parques realicen publicaciones de síntesis a medio camino entre el ámbito científico y la divulgación, en un marco más cercano al ocio y a las comunidades locales que al de la gestión del paisaje y el patrimonio predominante en Europa. Así, para Barrett y Copping (2005) son “paisajes vivientes a gran escala, donde comunidades y residentes se han unido alrededor de una visión común de su patrimonio compartido" (p. 1), mientras para Bray (1988) son:

Entornos urbanos y regionales multifuncionales con una coherencia o sentido de lugar distintivo basado en factores como ríos, lagos, sistemas de transporte y patrimonio cultural. Han sido llamados "parques asociativos" [partnerships] por la diversidad de participantes (incluyendo propietarios privados, organizaciones sin ánimo de lucro y múltiples unidades de agencias y gobiernos) involucrados en el planeamiento de los distintos objetivos de preservación, recreación, educación y desarrollo sostenible. Los parques exitosos mantienen a los residentes al frente en cuestiones de propiedad, control y celebración (pár. 190).

Finalmente, el NPs (2003) los define como:

Lugares designados por el Congreso de los EE.UU. donde recursos naturales, culturales, recreativos e históricos se combinan para formar un paisaje coherente y distintivo a nivel nacional, surgido de patrones de actividad humana configurada por la geografía. Estas áreas cuentan historias relevantes sobre nuestra nación y 
representan la experiencia nacional tanto a través de elementos materiales como de las tradiciones que han evolucionado en paralelo (pár. 10).

He querido citar de modo extenso estas definiciones subrayando sus elementos clave, porque, pese a su variedad disciplinar y contextual, confieren en conjunto una idea de cómo funcionan los parques patrimoniales. Estos son vistos como instrumentos, proyectos, paisajes, iniciativas regionales o museos que parten del binomio territorio-patrimonio de cara a la transición hacia un nuevo modelo socioeconómico basado en el ocio y el turismo. Es decir, el elemento que se requiere valorar pasa a ser la identidad de un territorio y los grupos sociales que habitan en él, como patrimonios tangible e intangible, respectivamente. Así, se orientan no solo a la preservación del pasado o de la naturaleza, como los espacios protegidos tradicionales, sino a la proyección del futuro a través de la reorganización de un espacio y un grupo social enraizado en el mismo.

En Europa, esta dinámica se plantea en relación con los conceptos de museo y paisaje, como la gestión de unos recursos patrimoniales encuadrados en un espacio de cara a la reactivación económica de áreas deprimidas. Las definiciones estadounidenses enfatizan la necesidad de garantizar la participación de las comunidades locales y la preservación de su identidad específica, fomentando la "narración de historias". Especialmente en el caso norteamericano, la ordenación de los parques culturales está relacionada con cuestiones sociopolíticas: búsqueda de la cohesión de comunidades alrededor de la idea de patrimonio, política territorial encaminada a la mitigación de las consecuencias de la desindustrialización, búsqueda de legitimidad a través de la narración de eventos e historias nacionales, etcétera.

La particularidad latinoamericana deriva de la existencia de paisajes más vastos que los europeos, que obligan a incluir una gran variedad de recursos heterogéneos (Sabaté, 2010). Si bien en la región no ha surgido una concepción estandarizada del parque patrimonial como instrumento de gestión, como podría ser en los EE.UU., sí se reconoce la tendencia al papel central del patrimonio en la ordenación de paisajes culturales (Gaiotto \& Argollo Ferrão, 2008). Igualmente, la gran cantidad de estudios académicos e institucionales sobre paisajes culturales puestos en valor turístico apuntan en la dirección de un futuro incremento de este tipo de proyectos (Paes, 2009). Han surgido así multitud de itinerarios o corredores (Ramírez, 2011), como los Caminos del Inca (Tresserras, 2006), o el Camino del Gaucho (Pesci $\&$ Pesci, 2006). Este último se ha convertido en una ruta cultural internacional diseńada, donde se articulan diversos recursos culturales y servicios turísticos estructurados a partir de una historia. Otros paisajes culturales se están articulando como parques patrimoniales a partir de declaraciones de la Unesco, como la Quebrada de Humahuaca (Salleras, 2011), y alrededor de ejes temáticos específicos, como el vino en Colchagua, Chile (Muñoz, 2011), o Mendoza, Argentina (Moretti, 2008), áreas cafetaleras en Colombia (Sarmiento, 2012), o incluso a través de la creación de parques agrarios para valorizar recursos patrimoniales en Brasil (Martins \& Argollo, 2010). Podemos decir, por tanto, que cabe esperar desarrollos en el área en la dirección de los parques patrimoniales y una tendencia a la estandarización de las prácticas y las formas. 
Constitución y funcionamiento de los parques culturales

Los parques patrimoniales se definen igualmente por un particular "modo de proceder". El objetivo de este apartado es mostrar las formas más comunes, según las cuales se constituyen y comienzan a funcionar. Existe una tendencia a crear parques en torno a elementos lineales como ríos, canales o caminos históricos, ya que facilitan la narración de una historia y la ordenación del espacio (véase Bustamante $\&$ Ponce, 2004, para una categorización de parques patrimoniales de acuerdo con sus dimensiones, localización espacial y forma de gestión). Pese a que el estudio de la constitución de los parques patrimoniales muestra cómo cada uno de ellos responde a unas coordenadas determinadas, existen elementos comunes.

Las NHA estadounidenses suelen surgir de iniciativas locales, desde grupos de entusiastas del patrimonio a asociaciones de empresarios. Según Conzen y Wulfestieg (2001), los promotores habituales durante los años noventa eran organizaciones no gubernamentales (ONG), generalmente ecologistas. Una vez que el proyecto se pone en marcha, los grupos locales suelen buscar el apoyo técnico y financiero del NPs, de los estados y del Gobierno Federal, lo que les facilita la atracción de fondos privados. La gestión suele realizarse mediante modelos corporativos donde predominan cuestiones de gestión económica y legal sobre factores culturales, algo comprensible teniendo en cuenta que el NPS solo provee quince años de financiación a cada NHA.

En Europa, al igual que en Latinoamérica (Flores, 2005), la naturaleza y extensión de las áreas patrimoniales "varía de país a país, al igual que el nivel de apoyo estatal. Sin embargo, existen ejemplos de áreas patrimoniales en casi todos los países" (Frenchman, 2004 , p. 1). Pese a la variedad, predomina un modelo burocrático, según el cual los parques surgen de arriba hacia abajo, desde administraciones nacionales y regionales o universidades. En lugar de economistas o abogados como en el caso estadounidense, la presencia más destacada suele ser de arquitectos, ingenieros y planificadores territoriales y, en menor medida, de arqueólogos y geógrafos. En el caso de Cataluña, Sabaté (2004) considera los parques patrimoniales como una iniciativa originalmente académica, que posteriormente contó con el soporte de instituciones regionales y grupos locales. La participación local es fomentada dentro de los esquemas de gobierno específicos de cada parque. Por ejemplo, en el consejo de gobierno de los parques culturales de Aragón (España) hay representantes del gobierno regional, de la Universidad de Zaragoza y de administraciones y asociaciones locales de todo tipo.

El proceso de constitución de los parques suele discurrir en paralelo a su proyección espacial. Lo habitual es que se lleve a cabo un inventario patrimonial previo en la región delimitada y una clasificación de los recursos de acuerdo con su valor y tipología. Todo parque cultural exitoso ha de organizar estos recursos patrimoniales alrededor de una historia, siendo necesario que los planificadores tengan habilidades similares a las de un guionista, ya que "elaborar el proyecto de un parque patrimonial resulta equivalente a construir el guión de una película" (Sabaté, 2004, p. 23). Esta afirmación es una muestra clara de la importación de modelos estadounidenses en Europa. Como habíamos visto, en EE.UU. los parques funcionan como depositarios de la identidad nacional, y el propio NPS reconoce que han de narrar historias relacionadas con eventos y personajes clave dentro de una dimensión relacionada con el ocio. En Europa sigue imperando una visión museística 
vinculada a la divulgación científica, la preservación de una identidad territorial adscrita a un paisaje y el equilibrio ecológico. En consecuencia, el patrimonio suele integrarse en redes y rutas sin llegar a conformar una narrativa general o una "historia", funcionando el parque como una denominación "paraguas" bajo la que se agrupan museos, sitios arqueológicos, monumentos, paisajes, pueblos históricos, etcétera. Así, mientras las NHA estadounidenses son relativamente fáciles de asociar a una temática, los parques europeos amalgaman elementos heterogéneos que se solapan cronológica y temáticamente en el palimpsesto paisajístico.

\section{Planificación}

Analizar la proyección espacial de los parques patrimoniales es complicado, por la dificultad de acceso a sus planes directores, raramente publicados. La ordenación de un parque se basa en la identificación de cinco elementos esenciales:

a. Ámbito global y subámbitos del parque: áreas.

b. Recursos patrimoniales y servicios: hitos.

c. Puertas y accesos, centros de interpretación y museos: nodos.

d. Caminos que vinculan todo: itinerarios.

e. Límites visuales y administrativos de la intervención: bordes.

Hay que tener en cuenta que su propuesta es una proyección ideal con una clara adscripción cultural enraizada en la tradición norteamericana, que favorece el intervencionismo y el diseño sobre la preservación, de cara al refuerzo de la identidad del parque. Por ejemplo, se fomenta la exaltación de los límites del parque mediante el uso de vegetación o señalización que "nos permitan reconocer en todo momento si nos hallamos dentro o fuera de un determinado ámbito patrimonial” (Sabaté, 2004, p. 25). Esta voluntad de intervenir espacialmente deriva de la necesidad de narrar una historia y, por lo tanto, de reorganizar el espacio a su alrededor.

Esta concepción intervencionista predominante en EE.UU., implementada por grupos locales con un enfoque esencialmente patrimonialista/ecologista, es potencialmente conflictiva, ya que si bien estos grupos no persiguen la propiedad del suelo, sí tienden a buscar el control de sus usos con miras a la preservación. Los planificadores territoriales estadounidenses conocen bien el rechazo existente en las zonas rurales a nuevas formas de zonificación (Means, 1999), rechazo generalmente canalizado a través de las distintas asociaciones de defensa de la propiedad privada. Estas consideran que las NHA violan los principios de la nación estadounidense, frenan el desarrollo económico y permiten a grupos ecologistas controlar los usos del suelo (DeWeese, 2005). Burling considera que:

El legado que dejaremos a las generaciones futuras no será la preservación de nuestra Historia, sino la de una fachada ficticia que oculta la erosión de los derechos que animaron nuestra historia durante los dos primeros siglos de la república (citado en National Center for Public Policy Research, 2006, p. 3).

Se plantea entonces la cuestión de cómo ordenar el territorio a partir del concepto de patrimonio. Para unos, "patrimonio" representa la preservación ecológica y los 
vestigios materiales del pasado; para otros, un ideal abstracto que sustenta la identidad nacional y no puede ser reducido a una expresión material.

En la visión europea prima una idea de paisaje abierto, sin límites o puertas, y un grado de intervención mucho menor. La planificación de los parques se inscribe en un marco amplio de intervención burocratizado, con lo que se reduce considerablemente la conflictividad del asunto. Los parques implican más un cambio en las formas institucionales de entender el territorio y su planificación, que una transformación radical en los usos del suelo. Así, la creación de un parque suele apoyarse en planes de desarrollo rural multifuncional previos y fomentar la tendencia a utilizar planes territoriales integrados en lugar de sectoriales. Otras actuaciones habituales incluyen la implementación de planes extraurbanos de ordenación agrícola, ambiental y paisajística de cara al desarrollo de la producción típica y biológica, y la mejora de los espacios recreativos. También se favorece la creación de planes urbanísticos dirigidos a la preservación arquitectónica y el control del crecimiento.

Así, la ordenación territorial ha de tener en cuenta las nuevas formas de valorización territorial y el papel variable que pueden desempeñar las identidades y actitudes locales ante un proceso de transformación. Esto supone no solo articular las políticas de turismo, urbanismo, territorio y ambiente alrededor de la idea de patrimonio, sino implicar a distintas instituciones y agentes sociales en la planificación del territorio de cara a la consolidación del parque patrimonial. El paisaje cultural se convierte en un patrimonio que se debe gestionar de modo sostenible, para producir riqueza que a la vez repercuta en el aumento de su valor y en la calidad de vida de sus habitantes. Esta realidad supone una ruptura radical con las políticas territoriales previas propias del industrialismo, en las que la producción de riqueza pasaba por el consumo irreversible de gran parte de los recursos del territorio (Magnaghi, 2000).

\section{¿Parques temáticos o museos? Hacia la tematización del territorio}

Los parques patrimoniales pueden considerarse como tentativas desarrolladas a pequeña escala dentro de un contexto general que tiende a la hibridación entre las dimensiones del ocio y la cultura (Thrift, 2008) y a la tematización del territorio. La cuestión de los parques temáticos no ha de tomarse a la ligera, ya que ellos influencian cada vez más las formas de producir espacio en EE.UU. y en el mundo (Bryman, 1999). Las diferencias entre parques patrimoniales y temáticos son evidentes. Estos últimos son áreas corporativas que desarrollan una temática lúdica para obtener beneficio. A diferencia de los parques patrimoniales, los parques temáticos no pueden ser definidos por una identidad clara, dada la ausencia de memoria y sociedad (Augé, 2008), ni por la presentación de una narrativa con base científica.

Sin embargo, las consecuencias a largo plazo de la combinación turismo-patrimonio son impredecibles y, de hecho, algunas NHA estadounidenses se acercan a los parques temáticos al narrar historias abstractas y realizar reconstrucciones y recreaciones artificiales. También resulta imposible distinguir entre parque temático o patrimonial en lugares como Puy du Fou en Francia, donde cultura y ocio se encuentran intrínsecamente unidos (Martin \& Suaud, 1992). En estos casos, la ordenación territorial entra de lleno en una dimensión centrada en el ocio que no hace más que potenciar la racionalización del espacio y de las formas de producción 
y consumo (Ritzer \& Liska, 1997). En paralelo, se está produciendo una tendencia a la adaptación de los parques temáticos a culturas locales, la disminución de su grado de abstracción y tematización y la inclusión de narrativas científicas, exhibiciones etnográficas o espacios propiamente urbanos, como Celebration City. Especialmente en Asia, el fenómeno de los parques temáticos tiende así a acercarse a los parques patrimoniales, al hibridarse con la cultura y patrimonio locales (Jones, Robinett \& Zito, 1993).

Las fronteras entre ocio y cultura, natura y cultura o autenticidad y copia tienden así a difuminarse en un contexto donde los parques patrimoniales se encuentran siempre a mitad de camino entre una ordenación territorial preservacionista, quizá más arraigada en Europa y que los asimila a museos, y la intervencionista americana, que favorece una mayor tematización. Los parques patrimoniales han de situarse siempre de un modo u otro entre estos dos ideales abstractos y opuestos, articulando la dialéctica entre transformación y fijación del paisaje patrimonial. La cuestión esencial es cuándo y cómo dejar de producir paisaje para fijarlo como un bien territorial metacultural. En este sentido, compartimos las reflexiones de Rubio cuando afirma que esta dialéctica se suele articular en los parques más a través de "planteamientos de orden estructuralista que funcionales y sistémicos y se hace en clave de cultura urbana y no de cultura propiamente territorial local" (Rubio, 2008, p. 46).

\section{Conclusiones y discusión}

La ordenación territorial, entendida como las distintas formas a través de las que definimos cómo usar el espacio y gobernar el territorio, refleja siempre la ideología - o sistema de valores y creencias dominantes- específica de cada época (Gunder, 2010). La tesis sostenida en este trabajo es que los parques patrimoniales son expresión de la ideología contemporánea, al conjugar las dimensiones del ocio y la cultura a través del concepto de patrimonio. En paralelo, son una solución válida para los problemas territoriales que ha conllevado la transición a una economía posindustrial. Son también útiles a nivel burocrático, al permitir la creación de "islas" de planeamiento donde rigen normas específicas. En el ámbito del mercado, los parques facilitan la reinvención y tematización del territorio, lo que potencia los flujos de turismo e inversiones y la generación de marcas que adscriben una identidad distintiva y, por tanto, un valor simbólico ańadido a los productos locales. Por ello, el territorio sigue siendo objeto de una mirada economicista, pero esta vez en un contexto de localización y regionalización que se opone a lo global (Castells, 2000).

La cuestión identitaria hace que el territorio pase a ganar un valor abstracto. Este valor reside en la tríada comunidad-paisaje-proyecto, enfocada al refuerzo de la identidad local de cara a la generación de una situación heurística que repercuta positivamente a distintas escalas: autoestima de la comunidad, mejora de la imagen territorial, atracción turística e inversora, calidad ambiental, etcétera. Esto obliga a que la ordenación territorial tome en consideración aspectos afectivos y emocionales de las configuraciones socioterritoriales sobre las que actúa. La cuestión 
esencial de los parques patrimoniales no es, entonces, la sustitución de un modelo de ordenación sectorial por uno integral, sino un cambio en la forma de percibir el territorio que lleve al abandono de la dicotomía naturaleza/cultura. En este sentido, no compartimos con Magnaghi (2000) la idea de que este tipo de procesos territoriales suponga el fin de la producción de paisaje. Más bien potencia la generación de nuevas dinámicas de acuerdo con el contexto socioeconómico e ideológico contemporáneo que, a su modo, produce un nuevo paisaje.

Es necesario analizar específicamente cada parque sin dar por hecho que se trata de herramientas positivas al vincularse con grandes narrativas de "desarrollo rural local" o "protección patrimonial". Los parques no garantizan per se una ordenación territorial más democrática. De hecho, bajo muchas de estas iniciativas se esconden procesos de turistificación implementados desde entornos urbanos que suscitan el rechazo local. Entonces, la ordenación territorial de los parques patrimoniales no se puede seguir planteando de un modo desterritorializado como durante el industrialismo, sino que ha de vincularse a cuestiones sociales. Por un lado, necesita del conocimiento territorial que las ciencias sociales pueden producir, para llevar a cabo una proyección certera del mismo. Por otro, la ordenación posee un efecto performativo al generar un nuevo contexto socioeconómico en el que unos actores entran a formar parte del entramado territorial, mientras otros pierden importancia: un escenario que puede generar situaciones conflictivas, como hemos visto con el caso de las NHA norteamericanas.

Es importante tener en cuenta que existe una tendencia a que la mayor parte de las áreas protegidas (parques nacionales, naturales, etcétera) adopten globalmente un modelo de gestión y ordenación similar al de los parques patrimoniales. Esto se debe a la creciente vinculación de toda área protegida con el desarrollo económico y a la toma de conciencia entre planificadores e instituciones respecto de la imposibilidad de gestionar parques y reservas como islas con límites definidos. De hecho, la mayor parte de los parques en el mundo se enfrenta a amenazas que provienen del exterior de sus límites, cuestión imposible de resolver mediante una gestión unilateral del territorio. Así, según el exdirector del NPs, Denis Galvin:

El futuro de nuestros parques, su integridad ecológica y la calidad de sus emplazamientos históricos, dependen de nuestra capacidad de establecer acuerdos duraderos con propietarios y comunidades locales. Debemos sobrepasar las viejas situaciones de conflicto entre parques y tierras adyacentes, en una nueva era donde se reconocen los intereses compartidos de los parques y sus vecinos (Citado en Bray, 1994, párr. 14).

La gran variedad de articulaciones de los parques patrimoniales, su potencial influencia en configuraciones macroterritoriales y en la articulación de áreas protegidas hace que sea necesario convertirlos en objeto de investigación pormenorizada. Nuestro análisis ha procurado tender puentes entre la ordenación territorial y las ciencias sociales, de modo que estas puedan aportar un punto de vista complementario al de perspectivas técnicas y abrir así la puerta a un tema de estudio que no puede sino ser aprehendido desde la interdisciplinariedad. @EURE 


\section{Referencias bibliográficas}

Augé, M. (2008). Non-places: An introduction to supermodernity (John Howe, Trad.). Londres: Verso.

Barrett, B. \& Copping, S. (2005). National heritage areas: Developing a model for measuring success. Briefing paper prepared for the 2004 us/Iсомos International Symposium. Natchitoches, Louisiana.

Barilaro, C. (2006). The role of literary parks in the re-appropriation of the cultural identity of places. En P. Claval, M. P. Pagnini \& M. Scaini (Eds.), The cultural turn in geography. Part II: Landscape construction and cultural identity (pp. 101-106). Gorizia: Gorizia Campus, Universidad de Trieste. Disponible en http://hdl.handle.net/10077/840

Barrett, B. (2003). Roots for the National Heritage Area family tree. The George Wright Forum, 20(2), 41-49.

Bergdhal, E. (2005). Ecomuseo Bergslagen, un proyecto sueco de parque cultural. Identidades: Territorio, cultura, patrimonio, (1), 68-71. Diponible en http://upcommons.upc.edu/ revistes/handle/2099/1888

Birnbaum, A. \& Capelle, C. (Eds.). (1996). The Secretary of the Interior's standards for the treatment of historic properties: with guidelines for the treatment of cultural landscapes. Washington, D.C.: U.s. Department of Interior.

Bray, P. M. (1988). Possibility of parks unbounded. Environment, 6(2), 88-97. Disponible en http://www.braypapers.com/PP.html

Bray, P. M. (1994). The heritage area phenomenon: where it is coming from. Cultural Resource Management, 17(8), 34. Disponible en http://www.braypapers.com/heritage.html

Bryman, A. (1999). The disneyization of society. The Sociological Review, 47(1), 25-47. doi: 10.1111/1467-954X.00161

Bustamante, L. P. \& Ponce, C. P. (2004). Paisajes culturales: el parque patrimonial como instrumento de revalorización y revitalización del territorio. Theoria, 13, 9-24. Disponible en http://www.ubiobio.cl/theoria/v/v13/1.pdf

Casey, E. S. (1997). The fate of place: a philosophical history. Berkeley, CA: University of California Press.

Castells, M. (2000). La sociedad real. Madrid: Alianza.

Clavel-Lévêque, M.; Doukellis, P. \& Tirologos, G. (2002). Cultural landscapes: new strategies of preservation. Artículo presentado en conferencia Conservation of cultural heritage for sustainable development, Cracow, Polonia. Disponible en http://www.cyfronet. krakow.pl/ ncbratas/pdf/full_levaque.pdf

Conzen, M. P. (2001). Heritage Corridors und neuer Tourismus an historischen Kanalen in den UsA. Geographische Rundschau, 53(1), 49-55. Disponible en http://bit.ly/16t7ZYh

Conzen, M. P. \& Wulfestieg, B. M. (2001). Metropolitan Chicago's Regional Cultural Park: Assessing the development of the Illinois \& Michigan Canal National Heritage Corridor. Journal of Geography, 100(3), 111-117. doi: 0.1080/00221340108978426

Choay, F. (2007). Alegoría del patrimonio. Barcelona: Gustavo Gili.

Daly, J. (2003). Heritage areas: connecting people to their place and history. Forum Journal: Regional Heritage Areas: Connecting People to Places and History, 17(4), 5-12. Disponible en http://bit.ly/17RGQ67 
DeWeese, T. (2005). Peyton Knight's testimony on National Heritage Areas submitted to the U.S. Senate subcommittee on National Parks. Disponible en http://bit.ly/15edtXM

Doménech Reinoso, J. (2005). La superficie y lo invisible. Patrones en la transformación territorial de la Cuenca Central Asturiana 1890-1960 (Disertación doctoral no publicada). Universidad Politécnica de Cataluña, Barcelona.

Eugster, J. G. (2003). Evolution of the heritage areas movement. The George Wright Forum, 20(2), 50-59. Disponible en http://www.webpages.uidaho.edu/css501/images/Readings/NHA.pdf

Flores, J. A. V. (2005). Da paisagem imposta à paisagem desejada: a dimensão cultural como eixo referencial na recuperação de cenários degradados (Disertación doctoral no publicada). Universidade Federal de Santa Catarina, Santa Catarina.

Frenchman, D. (2004). International examples of the United States heritage area concept. Disponible en http://www.nps.gov/history/heritageareas/REP/intlexamples.pdf

Fumagalli, A. (2007). Bioeconomia e capitalismo cognitivo: verso un nuovo paradigma di accumulazione. Roma: Carocci.

Gaiotto, M. A. \& Argollo Ferrão, A. M. (2008). O patrimônio histórico-cultural como ordenador da paisagem na bacia do Baixo Sorocaba, regiāo do Médio Tietê, em São Paulo. Artículo presentado en el $3^{\text {er }}$ Congresso Luso Brasileiro para o Planejamento Urbano, Regional, Integrado e Sustentável, Santos, São Paulo. Disponible en http://www.argollo.org/artigos_texto/Pluris-Paper772.pdf

Gunder, M. (2010). Planning as the ideology of (neoliberal) space. Planning Theory, 9(4), 298314. doi: $10.1177 / 1473095210368878$

Henry, R. (2000, agosto). Dancing into being: The Tjapukai Aboriginal Cultural Park and the Laura Dance Festival. The Australian journal of anthropology, 11(2), 322-332. doi: 10.1111/j.1835-9310.2000.tb00047.x

Jones, C. B.; Robinett, J. \& Zito, T. (1993). The future role of theme parks in international tourism. En J. R. Brent Ritchie et al. (Eds.), World Travel and Tourism Review: Indicators, trends and issues (Vol. 3, pp. 144-150). Wallingford, Oхоn: САВ International.

Koolhaas, R. (2002, primavera). Junkspace. October (100), 175-190. doi: $10.1162 / 016228702320218457$

Lacosta Aragües, A. J. (1997). Aprovechamiento turístico y conservación del patrimonio natural y cultural en los parques naturales regionales franceses. El caso del parque de Lorena. Geographicalia, 35, 129-148. Disponible en http://dialnet.unirioja.es/servlet/ articulo?codigo $=59872$

Latour, B. (1992). Aramis, ou, L'amour des techniques. París: La Découverte.

Law, J. \& Mol, A. (Eds.) (2002). Complexities: social studies of knowledge practices. Durham: Duke University Press.

Magnaghi, A. (2000). II progetto locale. Torino: Bollati Boringhieri.

Marazzi, C. (2010). The violence of financial capitalism (Kristina Lebedeva \& Jason F. Mc Gimsey, Trad.). Cambridge, MA: Massachusetts Institute of Technology (міт).

Martin, J. C. \& Suaud, C. (1992). Le Puy du Fou. L'interminable réinvention du Paysan vendéen. Actes de la recherche en sciences sociales, 93, 21-37. doi: 10.3406/arss.1992.3015 
Martin, S. (2005). The current state of heritage areas research: challenges and opportunities. En J. G. Peden \& R. M. Schusteer (Eds.), Proceedings of the Northeastern Recreation Research Symposium (pp. 24-30). Newton Square, PA: U.s. Forest Service, Northeastern Reasearch Station. Disponible en http://www.fs.fed.us/ne/newtown_square/ publications/technical_reports/pdfs/2006/341\%20papers/copping341.pdf

Martins, L. M. \& Argollo, A. M. (2010). Arquitetura da produçáa rural: o parque agrário como uma possibilidade de valorização dos recursos patrimoniais para os perímetros irrigados do nordeste do Brasil. Labor \& Engenho, 4(1).

Means, M. (1999). Happy trails. Planning (Journal of the American Planning Association), 65(8).

Moretti Baldín, G. (2008). Historia, historiografía y gestión cultural del patrimonio vitivinícola de Mendoza, Argentina. Apuntes, 21(1), 114-131. Disponible en http://bit.ly/18xnjow

Muñoz Parra, C. (2011). Colchagua, un paisaje ordenándose en torno al vino. Cuadernos de Investigación Urbanistica (66), 15-33. Disponible en http://bit.ly/13XQwDE

Negri, A. \& Vercellone, C. (2007). Il rapporto capitale/lavoro nel capitalismo cognitivo. Posse. Disponible en http://halshs.archives-ouvertes.fr/halshs-00264147

Nelson, J. G. \& Sportza, L. M. (2000). Evolving protected area thought and practice. The George Wright Forum, 17(2), 59-69.

Oviedo, G. (2004). Áreas protegidas y pueblos indígenas. En S. Arguedas Mora, L. Castaño \& J. M. Rodríguez (Eds.), Lineamientos y herramientas para un manejo creativo de las áreas protegidas (pp. 206-247). San José, Costa Rica: Organización para Estudios Tropicales, Programa de Políticas y Ciencias Ambientales. Disponible en http://bit.ly/16MyiJ3

Paes, M. T. D. (2009). Patrimônio cultural, turismo e identidades territoriais: um olhar geográfico. En R. Bartolho (Ed.), Turismo de base comunitária-diversidade de olhares e experiências brasileiras (pp. 162-176). Rio de Janeiro: Letra e Imagem.

Pesci, R. \& Pesci, L. (2006). "Camino del Gaucho". Paisaje cultural y desarrollo sustentable. Un camino de oportunidades y cooperacion para el Mercosur. En F.I. Isla \& C.A Lasta (Eds.), Manual de manejo costero para la Provincia de Buenos Aires (pp. 205-227). Mar del Plata: Universidad Nacional de Mar del Plata.

Phillips, A. (2002). Management guidelines for IUCN category V protected areas: Protected landscapes/seascapes. Gland, Switzerland: International Union for Conservation of Nature and Natura Resources (IUCN). Disponible en http://bit.ly/12eB7Cg

Phillips, A. (2003). Turning ideas on their head. The George Wright Forum, 20(2), 8-32.

Ramírez, J. H. (2011). Los caminos del patrimonio. Rutas turísticas e itinerarios culturales. PASOS, 9(2), 225-237. Disponible en http://www.pasosonline.org/Publicados/9211/ PS0211_01.pdf

Ritzer, G. \& Liska, A. (1997). 'McDisneyization' and 'post-tourism'. En C. Rojek \& J. Urry (Eds.), Touring cultures: Transformations of travel and theory (pp. 96-109). Londres: Routledge.

Rubio Terrado, P. (2008). Desarrollo local y patrimonio cultural: el parque cultural de Albarracín. Geographicalia (53), 21-48. Disponible en http://dialnet.unirioja.es/ servlet/articulo?codigo $=2714065$

Rullani, E. (2006). Economia della conoscenza: creatività e valore nel capitalismo delle reti. Roma: Carocci. 
Rullani, E. (2009). Knowledge economy and local development: The evolution of industrial districts and the new role of 'urban networks'. Review of Economic Conditions in Italy, 2, 237-284.

Sabaté, J. (2004). Paisajes culturales. El patrimonio como recurso básico para un nuevo modelo de desarrollo, Urban 9, 8-29. Disponible en http://dialnet.unirioja.es/servlet/ articulo? codigo $=2850736$

Sabaté, J. (2009). Proyecto de parque patrimonial fluvial del Ter. En J. Busquets \& A. Cortina (Coords.), Gestión del paisaje. Manual de protección, gestión y ordenación del paisaje (pp.625-642). Barcelona: Ariel.

Sabaté, J. (2010). De la preservación del patrimonio a la ordenación del paisaje: intervenciones en paisajes culturales (Europa-Latinoamérica). Labor \& Engenho, 4(1), 10-25. Disponible en http://conpadre.org/L\&E/L\&E_v4_n1_2010/02_p10-25.pdf

Sabaté, J. \& Lista, A. (2001). Casos d'estudi europeus. En J. Sabaté \& M. Schuster (Eds.), Projectant l'eix del Llobregat. Paisatge cultural i desenvolupament regional (pp. 78-90). Barcelona: Universitat Politècnica de Catalunya-Massachusets Institute of Technology.

Salleras, L. (2011). Territorio, turismo y desarrollo sustentable en la Quebrada deHumahuaca: Paisaje y naturaleza al servicio de la práctica turística. Estudios y perspectivas en turismo, 20(5), 1123-1143. Disponible en http://dialnet.unirioja.es/servlet/articulo?codigo=3739678

Sarmiento Nova, J. M. (2012). Paisaje cultural cafetero del centro occidente de Colombia: un patrimonio vivo. Labor \& Engenho, 6(2), 8-15. Disponible en http://www.conpadre. org/L\&E/L\&E_v6_n2_2012/02_p8-15.pdf

Taylor, K. (2009). Cultural landscapes and Asia: reconciling international and Southeast Asian regional values. Landscape Research, 34(1), 7-31. doi: 10.1080/01426390802387513

Thrift, N. J. (2008). Non-representational theory: Space, politics, affect. Londres: Routledge.

Tresserras, J. (2006). Rutas e itinerarios culturales en Iberoamérica. Cuadernos de Patrimonio Cultural y Turismo, 15, 13-56. Disponible en http://www.conaculta.gob.mx/turismocultural/cuadernos/cuaderno15_1.php

Vincent, C. H. \& Whiteman, D. (2008). Heritage areas: Background, proposals, and current issues. Washington D.C.: U.s. Library of Congress.

Wang, Z. \& Sun, P. (2001). Heritage corridors. A comparatively new protection and conservation method of heritages. Journal of Chinese Landscape Architecture, 5 [artículo digital].

\section{Documentos oficiales}

Consejo de Europa. (2000). Convenio Europeo del Paisaje [s.n]. Florencia: Autor. Disponible en http://bit.ly/17wQ5IL

Conservation Foundation. (1985). National Parks for a New Generation: Visions, Realities, Prospects. Washington D.C.: Autor.

Gobierno de Aragón (1997). Ley 12/1997, de 3 de diciembre, de Parques Culturales de Aragón (1997). Boletín Oficial de Aragón, 143, 6292-6295. Disponible en http://bit. ly/11YfoPB

National Center for Public Policy Research. (2006). Statement of Peyton Knight concerning H.R. 5195. The Journey Through Hallowed Ground National Heritage Area Act of 2006 Before the U.S. House of Representatives Committee on Resources Subcommittee on National Parks, Recreation and Public Lands. Disponible en http://naturalresources.house.gov/ UploadedFiles/PeytonTestimony09.28.06.pdf 
National Park Service. (2003). National Heritage Area feasibility study guidelines. Disponible en http://www.nps.gov/history/heritageareas/FSGUIDE/feasibility_guide.html

Unesco - United Nations Educational, Scientific and Cultural Organization. (2005, 2 de febrero). Directrices Prácticas para la Aplicación de la Convención del Patrimonio Mundial. whc.05/2. Comité Intergubernamental de Protección del Patrimonio Mundial Cultural y Natural, Centro del Patrimonio Mundial. Publicación, edición y traducción financiadas por los Fondos Extra-presupuestarios Españoles en el marco del Convenio firmado entre el Reino de Espańa y el Centro de Patrimonio Mundial de la Unesco. Disponible en http://whc.unesco.org/archive/opguide05-es.pdf 